\title{
Quantum-Classical Limit of Quantum Correlation Functions
}

\author{
Alessandro Sergi ${ }^{1, *}$ and Raymond Kapral ${ }^{1,2,}$, \\ ${ }^{1}$ Chemical Physics Theory Group, Department of Chemistry, \\ University of Toronto, Toronto, ON M5S 3H6, Canada \\ ${ }^{2}$ Fritz-Haber-Institut der Max-Planck-Gesellschaft, Faradayweg 4-6, 14195 Berlin, Germany
}

(Dated: 11th November 2018)

\begin{abstract}
A quantum-classical limit of the canonical equilibrium time correlation function for a quantum system is derived. The quantum-classical limit for the dynamics is obtained for quantum systems comprising a subsystem of light particles in a bath of heavy quantum particles. In this limit the time evolution of operators is determined by a quantum-classical Liouville operator but the full equilibrium canonical statistical description of the initial condition is retained. The quantum-classical correlation function expressions derived here provide a way to simulate the transport properties of quantum systems using quantum-classical surface-hopping dynamics combined with sampling schemes for the quantum equilibrium structure of both the subsystem of interest and its environment.
\end{abstract}

\section{INTRODUCTION}

The dynamical properties of systems close to equilibrium may be described in terms of equilibrium time correlation functions of dynamical variables or operators. For a quantum system with Hamiltonian $\hat{H}$ at temperature $T$ with volume $V$, linear response theory shows that the time correlation function of two operators $\hat{A}$ and $\hat{B}$, needed to obtain transport properties, has the Kubo transformed form [1, 2],

$$
\begin{aligned}
C_{A B}(t ; \beta) & =\frac{1}{\beta} \int_{0}^{\beta} d \lambda \operatorname{Tr} \hat{A}(t) e^{\lambda \hat{H}} \hat{B}^{\dagger} e^{-\lambda \hat{H}} \hat{\rho}_{e} \\
& =\frac{1}{\beta} \int_{0}^{\beta} d \lambda \frac{1}{Z_{Q}} \operatorname{Tr} \hat{B}^{\dagger} e^{\frac{i}{\hbar} t_{1}^{*} \hat{H}} \hat{A} e^{-\frac{i}{\hbar} t_{2} \hat{H}}
\end{aligned}
$$

where $\beta=\left(k_{B} T\right)^{-1}, \hat{\rho}_{e}=Z_{Q}^{-1} e^{-\beta \hat{H}}$ is the quantum canonical equilibrium density operator, $Z_{Q}=\operatorname{Tr} e^{-\beta \hat{H}}$ is the canonical partition function and, in the second line, $t_{1}=t-i \hbar(\beta-\lambda)$ and $t_{2}=t-i \hbar \lambda$. The evolution of the operator 
$\hat{A}(t)$ is given by the solution of the Heisenberg equation of motion, $d \hat{A}(t) / d t=\frac{i}{\hbar}[\hat{H}, \hat{A}(t)]$, where the square brackets denote the commutator.

While such correlation functions provide information on the transport properties of the system, their direct computation for condensed phase systems is not feasible due to our inability to simulate the quantum mechanical evolution equations for systems with a large number of degrees of freedom. While approximate schemes have been devised to treat quantum many body dynamics, for example, quantum mode coupling methods have proved useful in the calculation of collective modes for some applications [3], we are primarily concerned with methods that approximate the full many body evolution of the microscopic degrees of freedom. In many circumstances only a few degrees of freedom need to be treated quantum mechanically (quantum subsystem) while the remainder of the system with which they interact can be treated classically (classical bath) to a good approximation. Examples where such a description is appropriate include proton and electron transfer processes occurring in solvents or other chemical environments composed of heavy atoms. Quantum-classical methods have been reviewed by Egorov et al. [4] and one form of a quantum-classical approximation has been assessed in the weak coupling limit where there is no feedback between the quantum and classical subsystems. Although it is difficult to determine transport properties such as the reaction rate constant from the full quantum time correlation function when the entire system is treated quantum mechanically, methods are being developed to carry out such calculations. [5] Mixed quantum-classical methods also provide a route to carry out nonadiabatic rate calculations.

A number of schemes have been proposed for carrying out quantum dynamics in classical environments. 6 , 7, 8 , $9,10,11,12,13$ We focus on approaches where the evolution is described by a quantum-classical Liouville equation. 14, 15, 16, 17, 18, 19, 20, 21] For a quantum system coupled to a classical environment it is possible to derive an evolution equation for dynamical variables or operators (or the density matrix) by an expansion in a small parameter that characterizes the mass ratio of the light and heavy particles in the system. The quantum-classical analog of the Heisenberg equation of motion is, 21]

$$
\begin{aligned}
\frac{d}{d t} \hat{A}_{W}(X, t) & =\frac{i}{\hbar}\left[\hat{H}_{W}, \hat{A}_{W}(t)\right]-\frac{1}{2}\left(\left\{\hat{H}_{W}, \hat{A}_{W}(t)\right\}-\left\{\hat{A}_{W}(t), \hat{H}_{W}\right\}\right) \\
& =i \hat{\mathcal{L}} \hat{A}_{W}(X, t) .
\end{aligned}
$$

Here $\hat{A}_{W}(X, t)$ is the partial Wigner representation 21, 22] of a quantum operator; it is still an operator in the Hilbert space of the quantum subsystem but a function of the phase space coordinates $X=(R, P)$ of the classical bath. In this equation $\{\cdots, \cdots\}$ is the Poisson bracket and $\hat{\mathcal{L}}$ is the quantum-classical Liouville operator. A few features of quantum-classical Liouville dynamics are worth noting. This equation of motion includes feedback between the 
classical and quantum degrees of freedom. The environmental dynamics is fully classical only in the absence of coupling to the quantum subsystem. In the presence of coupling the environmental evolution cannot be described by Newtonian dynamics, although the simulation of the quantum-classical evolution can be formulated in terms of classical trajectory segments. 21] For harmonic environmental potentials with bilinear coupling to the quantum subsystem the evolution is equivalent to the fully quantum mechanical evolution of the entire system. Quantumclassical simulations of the spin-boson model are in accord with the numerically exact quantum results 23] and have been used to test quantum-classical simulation algorithms. 24, 25]

Equation (2) is valid in any basis and an especially convenient basis for simulating the evolution by surface-hopping schemes is the adiabatic basis, $\left\{\mid \alpha_{1} ; R>\right\}$, the set of eigenstates of the quantum subsystem Hamiltonian in the presence of fixed classical particles. In this case the matrix elements of an operator $A_{W}^{\alpha_{1} \alpha_{1}^{\prime}}(X, t)=<\alpha_{1} ; R\left|\hat{A}_{W}(X, t)\right| \alpha_{1}^{\prime} ; R>$ satisfy

$$
\frac{d}{d t} A_{W}^{\alpha_{1} \alpha_{1}^{\prime}}(X, t)=i \sum_{\beta_{1} \beta_{1}^{\prime}} \mathcal{L}_{\alpha_{1} \alpha_{1}^{\prime} \beta_{1} \beta_{1}^{\prime}} A_{W}^{\beta_{1} \beta_{1}^{\prime}}(X, t),
$$

where $\mathcal{L}_{\alpha_{1} \alpha_{1}^{\prime} \beta_{1} \beta_{1}^{\prime}}$ denotes the representation of the quantum-classical Liouville operator in the adiabatic basis. 21] This equation may be solved using surface-hopping schemes that combine a probabilistic description of the quantum transitions interspersed with classical evolution trajectory segments. 24, 25, 26, 27, 28] Although further algorithm development is needed to carry the simulations to arbitrarily long times, the quantum-classical evolution is not a short time approximation to full quantum evolution. Rather, it is an approximation to the full quantum evolution for arbitrary times since the quantum-classical evolution is derived at the level of the Liouville operator and not the quantum propagator. Given the evolution equation (2) (and the corresponding quantum-classical Liouville equation for the density matrix) one may construct a statistical mechanics of quantum-classical systems [29, 30] and compute transport properties such as chemical rate constants [31] from the correlation functions obtained from this analysis.

In this article we consider another route to determine quantum-classical correlation functions for transport properties. We begin with the full quantum mechanical expression for the time correlation function (Eq. (1)) and take the limit where the dynamics is determined by quantum-classical evolution equations for the spectral density that enters the correlation function expression. While the calculations leading to our expression for the correlation function are somewhat lengthy, the final result has a simple structure:

$$
\begin{aligned}
C_{A B}(t ; \beta)= & \sum_{\beta_{1}^{\prime} \beta_{1} \beta_{2}^{\prime} \beta_{2}} \int d X_{1} d X_{2} B_{W}^{\dagger \beta_{1} \beta_{1}^{\prime}}\left(X_{1}, \frac{t}{2}\right) \\
& \times A_{W}^{\beta_{2} \beta_{2}^{\prime}}\left(X_{2},-\frac{t}{2}\right) \bar{W}^{\beta_{1}^{\prime} \beta_{1} \beta_{2}^{\prime} \beta_{2}}\left(X_{1}, X_{2} ; \beta\right) .
\end{aligned}
$$


This expression for the time correlation function retains the full quantum statistical character of the initial condition through the spectral density function $\bar{W}$ (Eq. (40) below) but the forward and backward time evolution of the

operators $B_{W}^{\dagger \beta_{1} \beta_{1}^{\prime}}$ and $A_{W}^{\beta_{2} \beta_{2}^{\prime}}$, respectively, is given by the solutions of the quantum-classical evolution equation (3). Consequently, one may combine algorithms for determining quantum equilibrium properties with surface-hopping algorithms for quantum-classical evolution to estimate the value of the correlation function. Quantum effects enter in all orders in this expression for the correlation function. In addition to the fact that the initial value of the spectral density contains the full quantum equilibrium statistics, since the quantum-classical Liouville operator appears in the exponent in the propagator, the quantum-classical propagator contains all orders of $\hbar$, albeit in an approximate fashion.

The outline of the paper is as follows: In Sec. II we construct the partial Wigner representation of the quantum time correlation function and obtain expressions for the spectral density and its matrix elements in an adiabatic basis. In Sec. III we derive a quantum-classical evolution equation for the matrix elements of the spectral density and establish a connection to quantum-classical Liouville evolution. The results of these two sections are used in Sec. IV to obtain Eq. (4). In Sec. $\nabla$ we analyze the initial value of the spectral density in the high temperature limit. The conclusions of the study are given in Sec. $\mathbb{\nabla}$ ] while additional details of the calculations are presented in the Appendices.

\section{PARTIAL WIGNER REPRESENTATION OF QUANTUM CORRELATION FUNCTION}

We consider quantum systems whose degrees of freedom can be partitioned into two subsets corresponding to light (mass $m$ ) and heavy (mass $M$ ) particles, respectively. We use small and capital letters to denote operators for phase points in the light and heavy mass subsystems, respectively. In this notation the Hamiltonian operator for the entire system is the sum of the kinetic energy operators or the two subsystems and the potential energy of the entire system, $\hat{H}=\hat{P}^{2} / 2 M+\hat{p}^{2} / 2 m+\hat{V}(\hat{q}, \hat{Q})$.

We are interested in the limit where the dynamics of the heavy particle subsystem is treated classically and the light particle subsystem retains its full quantum character. To this end it is convenient to take a partial Wigner transform of the heavy degrees of freedom and represent the light degrees of freedom in some suitable basis.

In order to carry out this program we begin with the quantum mechanical Kubo transformed correlation function and write the trace over the heavy subsystem degrees of freedom in the second line of Eq. (10) using a $\{Q\}$ coordinate 
representation,

$$
C_{A B}(t ; \beta)=\frac{1}{\beta} \int_{0}^{\beta} d \lambda \frac{1}{Z_{Q}} \operatorname{Tr}^{\prime} \int \prod_{i=1}^{4} d Q_{i}<Q_{1}\left|\hat{B}^{\dagger}\right| Q_{2}><Q_{2}\left|e^{\frac{i}{\hbar} t_{1}^{*} \hat{H}}\right| Q_{3}><Q_{3}|\hat{A}| Q_{4}><Q_{4}\left|e^{-\frac{i}{\hbar} t_{2} \hat{H}}\right| Q_{1}>
$$

The prime in $\operatorname{Tr}^{\prime}$ refers to the fact that the trace is now only over the light particle subsystem degrees of freedom. Making use of the change of variables, $Q_{1}=R_{1}-Z_{1} / 2, Q_{2}=R_{1}+Z_{1} / 2, Q_{3}=R_{2}-Z_{2} / 2$ and $Q_{4}=R_{2}+Z_{2} / 2$, this equation may be written in the equivalent form,

$$
\begin{aligned}
C_{A B}(t ; \beta)= & \frac{1}{\beta} \int_{0}^{\beta} d \lambda \frac{1}{Z_{Q}} \operatorname{Tr}^{\prime} \int \prod_{i=1}^{2} d R_{i} d Z_{i}<R_{1}-\frac{Z_{1}}{2}\left|\hat{B}^{\dagger}\right| R_{1}+\frac{Z_{1}}{2}><R_{1}+\frac{Z_{1}}{2}\left|e^{\frac{i}{\hbar} t_{1}^{*} \hat{H}}\right| R_{2}-\frac{Z_{2}}{2}> \\
& \times<R_{2}-\frac{Z_{2}}{2}|\hat{A}| R_{2}+\frac{Z_{2}}{2}><R_{2}+\frac{Z_{2}}{2}\left|e^{-\frac{i}{\hbar} t_{2} \hat{H}}\right| R_{1}-\frac{Z_{1}}{2}>.
\end{aligned}
$$

The next step in the calculation is to replace the coordinate space matrix elements of the operators with their representation in terms of Wigner transformed quantities. The partial Wigner transform of an operator is defined by [21, 22$]$

$$
\hat{A}_{W}\left(R_{2}, P_{2}\right)=\int d Z_{2} e^{\frac{i}{\hbar} P_{2} \cdot Z_{2}}\left\langle R_{2}-\frac{Z_{2}}{2}|\hat{A}| R_{2}+\frac{Z_{2}}{2}\right\rangle
$$

while the inverse transform is

$$
\left\langle R_{2}-\frac{Z_{2}}{2}|\hat{A}| R_{2}+\frac{Z_{2}}{2}\right\rangle=\frac{1}{(2 \pi \hbar)^{\nu_{h}}} \int d P_{2} e^{-\frac{i}{\hbar} P_{2} \cdot Z_{2}} \hat{A}_{W}\left(R_{2}, P_{2}\right)
$$

Here $\nu_{h}$ is the dimension of the heavy mass subsystem. The partially Wigner transformed operator $\hat{A}_{W}\left(X_{2}\right)$ is a function of the phase space coordinates $X_{2} \equiv\left(R_{2}, P_{2}\right)$ and an operator in the Hilbert space of the quantum subsystem. It is convenient to consider a representation of such operators in basis of eigenfunctions. In this paper we use an adiabatic basis since, through this representation, we can make connection with surface-hopping dynamics. The partial Wigner transform of the Hamiltonian $\hat{H}$ is $\hat{H}_{W}=P^{2} / 2 M+\hat{p}^{2} / 2 m+\hat{V}_{W}(\hat{q}, R) \equiv P^{2} / 2 M+\hat{h}_{W}(R)$. The last equality defines the Hamiltonian $\hat{h}_{W}(R)$ for the light mass subsystem in the presence of fixed particles of the heavy mass subsystem. The adiabatic basis is determined from the solutions of the eigenvalue problem, $\hat{h}_{W}(R)\left|\alpha ; R>=E_{\alpha}(R)\right| \alpha ; R>$. The adiabatic representation of $\hat{A}_{W}\left(X_{2}\right)$ is,

$$
\hat{A}_{W}\left(X_{2}\right)=\sum_{\alpha_{2} \alpha_{2}^{\prime}}\left|\alpha_{2} ; R_{2}>A_{W}^{\alpha_{2} \alpha_{2}^{\prime}}\left(X_{2}\right)<\alpha_{2}^{\prime} ; R_{2}\right|
$$

where $A_{W}^{\alpha_{2} \alpha_{2}^{\prime}}\left(X_{2}\right)=<\alpha_{2} ; R_{2}\left|\hat{A}_{W}\left(X_{2}\right)\right| \alpha_{2}^{\prime} ; R_{2}>$.

By inserting Eq. (91) into Eq. (8) we can express the coordinate representation of the operator $\hat{A}$ as

$$
<R_{2}-\frac{Z_{2}}{2}|\hat{A}| R_{2}+\frac{Z_{2}}{2}>=\frac{1}{(2 \pi \hbar)^{\nu_{h}}} \sum_{\alpha_{2} \alpha_{2}^{\prime}} \int d P_{2} e^{-\frac{i}{2} P_{2} \cdot Z_{2}}\left|\alpha_{2} ; R_{2}>A_{W}^{\alpha_{2} \alpha_{2}^{\prime}}\left(X_{2}\right)<\alpha_{2}^{\prime} ; R_{2}\right|
$$


Then, substituting the result into Eq. (6) (along with a similar representation of the $\hat{B}^{\dagger}$ operator), we obtain,

$$
\begin{aligned}
C_{A B}(t ; \beta) & =\frac{1}{\beta} \int_{0}^{\beta} d \lambda \sum_{\alpha_{1}, \alpha_{1}^{\prime}, \alpha_{2}, \alpha_{2}^{\prime}} \int \prod_{i=1}^{2} d X_{i} B_{W}^{\dagger \alpha_{1} \alpha_{1}^{\prime}}\left(X_{1}\right) A_{W}^{\alpha_{2} \alpha_{2}^{\prime}}\left(X_{2}\right) \\
& \times W^{\alpha_{1}^{\prime} \alpha_{1} \alpha_{2}^{\prime} \alpha_{2}}\left(X_{1}, X_{2}, t ; \beta, \lambda\right) .
\end{aligned}
$$

Here we defined the matrix elements of the spectral density by

$$
\begin{aligned}
W^{\alpha_{1}^{\prime} \alpha_{1} \alpha_{2}^{\prime} \alpha_{2}}\left(X_{1}, X_{2}, t ; \beta, \lambda\right)= & \frac{1}{Z_{Q}} \int \prod_{i=1}^{2} d Z_{i} e^{-\frac{i}{\hbar}\left(P_{1} \cdot Z_{1}+P_{2} \cdot Z_{2}\right)}<\alpha_{1}^{\prime} ; R_{1}\left|<R_{1}+\frac{Z_{1}}{2}\right| e^{\frac{i}{\hbar} t_{1}^{*} \hat{H}}\left|R_{2}-\frac{Z_{2}}{2}>\right| \alpha_{2} ; R_{2}> \\
& \times<\alpha_{2}^{\prime} ; R_{2}\left|<R_{2}+\frac{Z_{2}}{2}\right| e^{-\frac{i}{\hbar} t_{2} \hat{H}}\left|R_{1}-\frac{Z_{1}}{2}>\right| \alpha_{1} ; R_{1}>\frac{1}{(2 \pi \hbar)^{2 \nu_{h}}}
\end{aligned}
$$

Our task is now to find an evolution equation for $W^{\alpha_{1}^{\prime} \alpha_{1} \alpha_{2}^{\prime} \alpha_{2}}\left(X_{1}, X_{2}, t ; \beta, \lambda\right)$ in the mixed quantum-classical limit. Before doing this we observe that the expression for the quantum correlation function in the partial Wigner representation is equivalent to an expression involving full Wigner transforms of the operators.

\section{A. Relation to full Wigner representation}

Since the correlation function is independent of the representation we choose for the light and heavy mass subsystems, we may also represent the light mass subsystem in terms of a Wigner transform instead of a set of basis functions. To establish the connection between these two forms of the correlation function we note that the full Wigner transform of the the operator $\hat{A}$ is given by

$$
\begin{aligned}
A_{W}\left(x_{2}, X_{2}\right) & =\int d z_{2} e^{\frac{i}{\hbar} p_{2} \cdot z_{2}}<r_{2}-\frac{z_{2}}{2}\left|\hat{A}_{W}\left(X_{2}\right)\right| r_{2}+\frac{z_{2}}{2}> \\
& =\sum_{\alpha_{2} \alpha_{2}^{\prime}} \int d z_{2} e^{\frac{i}{\hbar} p_{2} \cdot z_{2}} \phi_{\alpha_{2}}\left(r_{2}-\frac{z_{2}}{2} ; R_{2}\right) A_{W}^{\alpha_{2} \alpha_{2}^{\prime}}\left(X_{2}\right) \phi_{\alpha_{2}^{\prime *}}\left(r_{2}+\frac{z_{2}}{2} ; R_{2}\right),
\end{aligned}
$$

where $x_{2}=\left(r_{2}, p_{2}\right)$ and $\phi_{\alpha_{2}}\left(r_{2} ; R_{2}\right)=<r_{2} \mid \alpha_{2} ; R_{2}>$. We have used Eq. (9) to write the second line of this equation. The inverse of this expression is

$$
A_{W}^{\alpha_{2} \alpha_{2}^{\prime}}\left(X_{2}\right)=\frac{1}{(2 \pi \hbar)^{\nu_{\ell}}} \int d p_{2} d\left(r_{2}-\frac{z_{2}}{2}\right) d\left(r_{2}+\frac{z_{2}}{2}\right) e^{-\frac{i}{\hbar} p_{2} \cdot z_{2}} A_{W}\left(x_{2}, X_{2}\right) \phi_{\alpha_{2}}^{*}\left(r_{2}-\frac{z_{2}}{2} ; R_{2}\right) \phi_{\alpha_{2}^{\prime}}\left(r_{2}+\frac{z_{2}}{2} ; R_{2}\right),
$$

where $\nu_{\ell}$ is the dimension of the light mass subsystem. Inserting this expression for $A_{W}^{\alpha_{2} \alpha_{2}^{\prime}}\left(X_{2}\right)$ (and the analogous expression for $\left.B_{W}^{\dagger \alpha_{1} \alpha_{1}^{\prime}}\left(X_{1}\right)\right)$ into Eq. (11) we find

$$
\begin{aligned}
C_{A B}(t, \beta)= & \frac{1}{\beta} \int_{0}^{\beta} d \lambda \int \prod_{i=1}^{2} d x_{i} d X_{i} B_{W}^{\dagger}\left(x_{1}, X_{1}\right) A_{W}\left(x_{2}, X_{2}\right) \\
& \times W\left(x_{1}, X_{1}, x_{2}, X_{2} ; t, \beta, \lambda\right)
\end{aligned}
$$


where

$$
\begin{aligned}
W\left(x_{1}, x_{2}, X_{1}, X_{2}, t ; \beta, \lambda\right)= & \sum_{\alpha_{1}^{\prime} \alpha_{1} \alpha_{2}^{\prime} \alpha_{2}} \int \prod_{i=1}^{2} d z_{i} e^{-\frac{i}{\hbar}\left(p_{1} \cdot z_{1}+p_{2} \cdot z_{2}\right)} \phi_{\alpha_{1}^{\prime}}\left(r_{1}+\frac{z_{1}}{2} ; R_{1}\right) \phi_{\alpha_{1}}^{*}\left(r_{1}-\frac{z_{1}}{2} ; R_{1}\right) \\
& \times \phi_{\alpha_{2}^{\prime}}\left(r_{2}+\frac{z_{2}}{2} ; R_{2}\right) \phi_{\alpha_{2}}^{*}\left(r_{2}-\frac{z_{2}}{2} ; R_{2}\right) W^{\alpha_{1}^{\prime} \alpha_{1} \alpha_{2}^{\prime} \alpha_{2}}\left(X_{1}, X_{2}, t ; \beta, \lambda\right) \frac{1}{(2 \pi \hbar)^{2 \nu_{l}}} .
\end{aligned}
$$

Using the definition of the matrix elements of $W$ in Eq. (12) and performing the sums on states we may write this equation in the equivalent form,

$$
\begin{aligned}
W\left(x_{1}, x_{2}, X_{1}, X_{2}, t ; \beta, \lambda\right)= & \frac{1}{Z_{Q}} \int \prod_{i=1}^{2} d z_{i} d Z_{i} e^{-\frac{i}{\hbar}\left(p_{1} \cdot z_{1}+p_{2} \cdot z_{2}\right)} e^{-\frac{i}{\hbar}\left(P_{1} \cdot Z_{1}+P_{2} \cdot Z_{2}\right)} \\
& \times<r_{1}+\frac{z_{1}}{2}\left|<R_{1}+\frac{Z_{1}}{2}\right| e^{\frac{i}{\hbar} t_{1}^{*} \hat{H}}\left|R_{2}-\frac{Z_{2}}{2}>\right| r_{2}-\frac{z_{2}}{2}> \\
& \times<r_{2}+\frac{z_{2}}{2}\left|<R_{2}+\frac{Z_{2}}{2}\right| e^{-\frac{i}{\hbar} t_{2} \hat{H}}\left|R_{1}-\frac{Z_{1}}{2}>\right| r_{1}-\frac{z_{1}}{2}>\frac{1}{(2 \pi \hbar)^{2 \nu}},
\end{aligned}
$$

where $\nu=\nu_{\ell}+\nu_{h}$. Equation (17) gives the spectral density in the full Wigner representation while Eq. (16) relates this quantity to its matrix elements in the light mass subsystem basis. In particular, letting $\mathbf{W}$ be a (super) matrix whose elements are $W^{\alpha_{1}^{\prime} \alpha_{1} \alpha_{2}^{\prime} \alpha_{2}}$, Eq. (16) may be written formally as

$$
W=\mathcal{T} \circ \mathbf{W},
$$

where $\mathcal{T}$ is the transformation specified by Eq. (16).

For future use, we note that the inverse of this expression is given by

$$
\begin{aligned}
W^{\alpha_{1}^{\prime} \alpha_{1} \alpha_{2}^{\prime} \alpha_{2}}\left(X_{1}, X_{2}, t ; \beta, \lambda\right)= & \int \prod_{i=1}^{2} d x_{i} d z_{i} e^{\frac{i}{\hbar}\left(p_{1} \cdot z_{1}+p_{2} \cdot z_{2}\right)} \phi_{\alpha_{1}}\left(r_{1}-\frac{z_{1}}{2} ; R_{1}\right) \phi_{\alpha_{1}^{\prime}}^{*}\left(r_{1}+\frac{z_{1}}{2} ; R_{1}\right) \\
& \times \phi_{\alpha_{2}}\left(r_{2}-\frac{z_{2}}{2} ; R_{2}\right) \phi_{\alpha_{2}^{\prime}}^{*}\left(r_{2}+\frac{z_{2}}{2} ; R_{2}\right) W\left(x_{1}, x_{2}, X_{1}, X_{2}, t ; \beta, \lambda\right),
\end{aligned}
$$

as can be verified by substituting Eq. (17) into Eq. (19) and performing the integrals. Like Eq. (16), Eq. (19) gives a relation between $W$ and its matrix elements but now in the opposite direction, relating $W^{\alpha_{1}^{\prime} \alpha_{1} \alpha_{2}^{\prime} \alpha_{2}}$ to $W$. Using a formal notation like that in Eq. (18), we can write Eq. (19) as

$$
\mathbf{W}=\mathcal{T}^{-1} \circ W
$$

which defines $\mathcal{T}^{-1}$, the inverse of $\mathcal{T}$.

\section{QUANTUM-CLASSICAL EVOLUTION EQUATION FOR SPECTRAL DENSITY}

The quantum-classical evolution equation for matrix elements of the spectral density $W^{\alpha_{1}^{\prime} \alpha_{1} \alpha_{2}^{\prime} \alpha_{2}}\left(X_{1}, X_{2}, t ; \beta, \lambda\right)$ can be obtained using various routes. In this paper we first derive a quantum-classical evolution equation for the spectral 
density $W\left(x_{1}, x_{2}, X_{1}, X_{2}, t ; \beta, \lambda\right)$ in the full Wigner representation. We then change to an adiabatic basis representation of the quantum subsystem to obtain our final result for the evolution equation for $W^{\alpha_{1}^{\prime} \alpha_{1} \alpha_{2}^{\prime} \alpha_{2}}\left(X_{1}, X_{2}, t ; \beta, \lambda\right)$.

\section{A. Evolution of $W$ in the full Wigner representation}

The evolution equation for $W\left(x_{1}, x_{2}, X_{1}, X_{2}, t ; \beta, \lambda\right)$ can be obtained by differentiating its definition in Eq. (17) with respect to time and then inserting complete sets of coordinate states to obtain a closed equation in $W$. The result was obtained earlier by Filinov et al. [32] and, for our composite system, is given by

$$
\begin{aligned}
\frac{\partial}{\partial t} W\left(x_{1}, x_{2}, X_{1}, X_{2}, t ; \beta, \lambda\right) & =-\frac{1}{2}\left(i L_{1}^{(0)}\left(x_{1}, X_{1}\right)-i L_{2}^{(0)}\left(x_{2}, X_{2}\right)\right) W\left(x_{1}, x_{2}, X_{1}, X_{2}, t ; \beta, \lambda\right) \\
& +\frac{1}{2} \int \prod_{i=1}^{2} d s_{i} d S_{i}\left(\omega_{1}\left(r_{1}, s_{1}, R_{1}, S_{1}\right) \delta\left(s_{2}\right) \delta\left(S_{2}\right)-\omega_{2}\left(r_{2}, s_{2}, R_{2}, S_{2}\right) \delta\left(s_{1}\right) \delta\left(S_{1}\right)\right) \\
& \times W\left(x_{1}-\pi_{1}, X_{1}-\Pi_{1}, x_{2}-\pi_{2}, X_{2}-\Pi_{2}, t ; \beta, \lambda\right) .
\end{aligned}
$$

Here we have introduced the notation $\pi_{i}=\left(0, s_{i}\right), \Pi_{i}=\left(0, S_{i}\right)$. The classical free streaming Liouville operators are

$$
i L_{i}^{(0)}\left(x_{i}, X_{i}\right)=\frac{p_{i}}{m} \cdot \frac{\partial}{\partial q_{i}}+\frac{P_{i}}{M} \cdot \frac{\partial}{\partial R_{i}},
$$

for $(i=1,2)$. The $\omega_{i}$ functions under the integral are defined by

$$
\begin{aligned}
\omega_{i}\left(r_{i}, R_{i}, s_{i}, S_{i}\right) & =\frac{2}{\hbar(\pi \hbar)^{\nu}} \int d \bar{r}_{i} d \bar{R}_{i} V\left(r_{i}-\bar{r}_{i}, R_{i}-\bar{R}_{i}\right) \\
& \times \sin \left(\frac{2}{\hbar} s_{i} \cdot \bar{r}_{i}+\frac{2}{\hbar} S_{i} \cdot \bar{R}_{i}\right) .
\end{aligned}
$$

\section{B. Scaled equation of motion}

In order to take the quantum-classical limit of Eq. (21), we consider systems for which the ratio between the light particle mass and the heavy particle mass is small, and employ the same mass scaling used in Ref. [21] to obtain the quantum-classical Liouville equation. One is naturally led to consider an expansion in the small parameter $\mu=(m / M)^{1 / 2}$ from the following arguments. Consider a unit of energy $\epsilon_{0}$, say the thermal energy $\epsilon_{0}=\beta^{-1}$, a unit of length $\lambda_{m}=\hbar / p_{m}$, where $p_{m}=\left(m \epsilon_{0}\right)^{1 / 2}$ is the unit of momentum of the light particles, a unit of time $t_{0}=\hbar \epsilon_{0}^{-1}$ and a unit of momentum for the heavy particles $P_{M}=\left(M \epsilon_{0}\right)^{1 / 2}$. These units may be used to scale the coordinates of the system so that the magnitude of the scaled momentum of the heavy particles, $P / P_{M}$, is of the same order of magnitude as that for the light particles, $p / p_{m}$. Only momenta are scaled by different factors; characteristic lengths are scaled by the light particle thermal de Broglie wavelength, $\lambda_{m}$. This is analogous to the scaling used to derive the equations of Brownian motion for a heavy particle in a bath of light particles. 
In the following, we denote scaled quantities with a prime; e.g., $r^{\prime}=r / \lambda_{m}, R^{\prime}=R / \lambda_{m}, p^{\prime}=p / p_{m}, P^{\prime}=P / P_{M}$, $t^{\prime}=t / t_{0}$, etc. The scaled version of the equation of motion for $W$ has the same form as Eq. (21) but with all quantities replaced by their primed dimensionless counterparts. The scaled operators and functions in this equation are

$$
i L_{i}^{(0) \prime}\left(x_{i}^{\prime}, X_{i}^{\prime}\right)=p_{i}^{\prime} \cdot \frac{\partial}{\partial q_{i}^{\prime}}+\mu P_{i}^{\prime} \cdot \frac{\partial}{\partial R_{i}^{\prime}}
$$

and

$$
\begin{aligned}
\omega_{i}^{\prime}\left(r_{i}^{\prime}, R_{i}^{\prime}, s_{i}^{\prime}, S_{i}^{\prime}\right) & =\frac{2}{\pi^{\nu}} \int d \bar{r}_{i}^{\prime} d \tilde{R}_{i}^{\prime} V^{\prime}\left(r_{i}^{\prime}-\bar{r}_{i}^{\prime}, R_{i}^{\prime}-\mu \tilde{R}_{i}^{\prime}\right) \\
& \times \sin \left(2 s_{i}^{\prime} \cdot \bar{r}_{i}^{\prime}+2 S_{i}^{\prime} \cdot \tilde{R}_{i}^{\prime}\right) .
\end{aligned}
$$

In writing the last line of the $\omega_{i}^{\prime}$ equation we have performed the change of variables $\tilde{R}_{1}=\mu^{-1} \bar{R}_{1}$ in the dummy variable in the integration in order to move the $\mu$ dependence from the sine factor to the potential, which is more convenient for taking the classical limit. We see that the classical free streaming evolution is linear in $\mu$ but the quantum kernel has all powers of $\mu$.

\section{Quantum-classical equation for $W$}

For $\mu<<1$ the quantum-classical limit is obtained expanding the evolution operator up to linear terms in $\mu$. Since momentum is related to the de Broglie wavelength of a particle, this procedure is equivalent to averaging out the short de Broglie oscillations of the heavy particles on the scale of the long de Broglie oscillations of the light particles.

The expansion of the evolution operator is obtained from the expansion of the interaction potential to linear order in the small parameter $\mu$,

$$
V^{\prime}\left(r_{i}^{\prime}-\bar{r}_{i}^{\prime}, R_{i}^{\prime}-\mu \tilde{R}_{i}^{\prime}\right)=V\left(r_{i}^{\prime}-\bar{r}_{i}^{\prime}, R_{i}^{\prime}\right)-\mu \tilde{R}_{i}^{\prime} \cdot \frac{\partial V^{\prime}\left(r_{i}^{\prime}-\bar{r}_{i}^{\prime}, R_{i}^{\prime}\right)}{\partial R_{i}^{\prime}}+\mathcal{O}\left(\mu^{2}\right)
$$

Inserting this expansion in Eq. (25), working out the integrals, substituting the result into the scaled version of Eq. (21) and finally going back to unscaled coordinates (the details are given in Appendix equation for $W$ in unscaled coordinates as

$$
\begin{aligned}
& \frac{\partial}{\partial t} W\left(x_{1}, x_{2}, X_{1}, X_{2}, t ; \beta, \lambda\right)=-\frac{1}{2}\left[i L_{1}^{(0)}\left(x_{1}\right)+i L_{1}\left(X_{1}\right)-i L_{2}^{(0)}\left(x_{2}\right)-i L_{2}\left(X_{2}\right)\right] W\left(x_{1}, x_{2}, X_{1}, X_{2}, t ; \beta, \lambda\right) \\
& \quad+\int d s_{1} d s_{2}\left\{\frac{1}{\hbar(\pi \hbar)^{\nu_{\ell}}} \int d \bar{r}\left[\delta\left(s_{2}\right) V\left(r_{1}-\bar{r}, R_{1}\right) \sin \left(\frac{2 s_{1} \cdot \bar{r}}{\hbar}\right)-\delta\left(s_{1}\right) V\left(r_{2}-\bar{r}, R_{2}\right) \sin \left(\frac{2 s_{2} \cdot \bar{r}}{\hbar}\right)\right]\right. \\
& \left.\quad+\frac{1}{2}\left[\delta\left(s_{2}\right) \Delta F_{1}\left(R_{1}, s_{1}\right) \cdot \frac{\partial}{\partial P_{1}}-\delta\left(s_{1}\right) \Delta F_{2}\left(R_{2}, s_{2}\right) \cdot \frac{\partial}{\partial P_{2}}\right]\right\} W\left(x_{1}-\pi_{1}, x_{2}-\pi_{2}, X_{1}, X_{2}, t ; \beta, \lambda\right) .
\end{aligned}
$$


Here we have defined full classical Liouville operator for the heavy mass subsystem as,

$$
i L_{i}\left(X_{i}\right)=\frac{P_{i}}{M} \cdot \frac{\partial}{\partial R_{i}}+F_{R_{i}} \cdot \frac{\partial}{\partial P_{i}}
$$

for $(i=1,2)$, where the force $F_{R_{i}}=-\partial V\left(r_{i}, R_{i}\right) / \partial R_{i}$. We have also introduced the quantity

$$
\Delta F_{i}\left(R_{i}, s_{i}\right)=-\frac{\partial}{\partial R_{i}}\left(V\left(r_{i}, R_{i}\right) \delta\left(s_{i}\right)-\frac{1}{(\pi \hbar)^{\nu_{\ell}}} \int d \bar{r} V\left(r_{i}-\bar{r}, R_{i}\right) \cos \left(\frac{2 s_{i} \bar{r}}{\hbar}\right)\right) .
$$

If the potential is decomposed into light and heavy mass subsystem potentials, $V_{\ell}\left(r_{i}\right)$ and $V_{h}\left(R_{i}\right)$, respectively, and their interaction potential $V_{c}\left(r_{i}, R_{i}\right)$ as $V=V_{\ell}+V_{h}+V_{c}$, it is easy to demonstrate that $\Delta F_{i}\left(R_{i}, s_{i}\right)$ depends only on the interaction potential.

The quantum-classical evolution equation (27) for $W$ can be written formally and compactly as

$$
\frac{\partial}{\partial t} W(t)=\frac{1}{2} K \circ W(t)
$$

where the operator $K$ is defined by comparison with Eq. (27). To simplify the notation, we have dropped the classical phase space arguments here and some of the following equations when confusion is unlikely to arise.

This quantum-classical evolution equation for the spectral density is not yet in a convenient form for simulation since the kernels that appear in this equation are highly oscillatory functions arising from the fact that a Wigner representation of the quantum degrees of freedom has been used. In the next subsection we re-introduce the adiabatic basis and obtain a form of the quantum-classical evolution equation that can be solved by surface-hopping schemes.

\section{Quantum-classical evolution equation for $W^{\alpha_{1}^{\prime} \alpha_{1} \alpha_{2}^{\prime} \alpha_{2}}$}

The operators $\mathcal{T}$ and $\mathcal{T}^{-1}$ can be used to convert Eq. (30) into an evolution equation for $\mathbf{W}$, the matrix elements of $W$. Acting from the left with $\mathcal{T}^{-1}$ on Eq. (30) and inserting unity in the form $W=\mathcal{T} \circ \mathcal{T}^{-1} \circ W=\mathcal{T} \circ \mathbf{W}$, we obtain,

$$
\begin{aligned}
\frac{\partial}{\partial t} \mathbf{W}(t) & =\frac{1}{2}\left(\mathcal{T}^{-1} \circ K \circ \mathcal{T}\right) \circ \mathbf{W}(t) \\
& \equiv \frac{1}{2} \mathcal{K} \circ \mathbf{W}(t),
\end{aligned}
$$

The transformed operator on right hand side of Eq. (31) can be calculated explicitly by a straightforward but lengthy calculation which is given in detail in Appendix $\mathbb{B}$ The result of this calculation is

$$
\frac{\partial}{\partial t} W^{\alpha_{1}^{\prime} \alpha_{1} \alpha_{2}^{\prime} \alpha_{2}}\left(X_{1}, X_{2}, t ; \beta, \lambda\right)=\frac{1}{2} \sum_{\beta_{1}^{\prime} \beta_{1} \beta_{2}^{\prime} \beta_{2}} \mathcal{K}_{\alpha_{1}^{\prime} \alpha_{1} \alpha_{2}^{\prime} \alpha_{2}, \beta_{1}^{\prime} \beta_{1} \beta_{2}^{\prime} \beta_{2}} W^{\beta_{1}^{\prime} \beta_{1} \beta_{2}^{\prime} \beta_{2}}\left(X_{1}, X_{2}, t ; \beta, \lambda\right)
$$




$$
\begin{aligned}
= & \frac{1}{2} \sum_{\beta_{1}^{\prime} \beta_{1} \beta_{2}^{\prime} \beta_{2}}\left(-i \mathcal{L}_{\alpha_{1}^{\prime} \alpha_{1}, \beta_{1}^{\prime} \beta_{1}}\left(X_{1}\right) \delta_{\alpha_{2}^{\prime} \beta_{2}^{\prime}} \delta_{\alpha_{2} \beta_{2}}+i \mathcal{L}_{\alpha_{2}^{\prime} \alpha_{2}, \beta_{2}^{\prime} \beta_{2}}\left(X_{2}\right) \delta_{\alpha_{1}^{\prime} \beta_{1}^{\prime}} \delta_{\alpha_{1} \beta_{1}}\right) \\
& \times W^{\beta_{1}^{\prime} \beta_{1} \beta_{2}^{\prime} \beta_{2}}\left(X_{1}, X_{2}, t ; \beta, \lambda\right) .
\end{aligned}
$$

We see that the apparently formidable evolution operator $\mathcal{K}$ for the matrix elements of the spectral density, which depends on eight quantum indices, takes a simple form consisting of a difference of two quantum-classical Liouville operators, each acting separately on the classical phase space variables and quantum indices with labels 1 and 2 . The quantum-classical Liouville operators are just those obtained in earlier derivations of the quantum-classical Liouville equation [21],

$$
i \mathcal{L}_{\alpha_{i}^{\prime} \alpha_{i}, \beta_{i}^{\prime} \beta_{i}}\left(X_{i}\right)=\left(i \omega_{\alpha_{i}^{\prime} \alpha_{i}}\left(R_{i}\right)+i L_{\alpha_{i}^{\prime} \alpha_{i}}\left(X_{i}\right)\right) \delta_{\alpha_{i}^{\prime} \beta_{i}^{\prime}} \delta_{\alpha_{i} \beta_{i}}-J_{\alpha_{i}^{\prime} \alpha_{i}, \beta_{i}^{\prime} \beta_{i}}\left(X_{i}\right)
$$

where $\omega_{\alpha \alpha^{\prime}}(R)=\left(E_{\alpha}(R)-E_{\alpha^{\prime}}(R)\right) / \hbar$ and

$$
i L_{\alpha_{i}^{\prime} \alpha_{i}}\left(X_{i}\right)=\frac{P_{i}}{M} \cdot \frac{\partial}{\partial R_{i}}+\frac{1}{2}\left(F_{W}^{\alpha_{i}^{\prime}}\left(R_{i}\right)+F_{W}^{\alpha_{i}}\left(R_{i}\right)\right) \cdot \frac{\partial}{\partial P_{i}},
$$

is the classical Liouville operator involving the mean of the Hellmann-Feynman forces where $F_{W}^{\alpha}=$ $-\left\langle\alpha ; R\left|\frac{\partial \hat{V}_{W}(\hat{q}, R)}{\partial R}\right| \alpha ; R\right\rangle=-\left\langle\alpha ; R\left|\frac{\partial \hat{H}_{W}(R)}{\partial R}\right| \alpha ; R\right\rangle$. Quantum transitions and bath momentum changes are described by

$$
\begin{aligned}
J_{\alpha_{i}^{\prime} \alpha_{i}, \beta_{i}^{\prime} \beta_{i}}\left(X_{i}\right)= & -\frac{P_{i}}{M} \cdot d_{\alpha_{i}^{\prime} \beta_{i}^{\prime}}\left(1+\frac{1}{2} S_{\alpha_{i}^{\prime} \beta_{i}^{\prime}}\left(R_{i}\right) \cdot \frac{\partial}{\partial P_{i}}\right) \delta_{\alpha_{i} \beta_{i}} \\
& -\frac{P_{i}}{M} \cdot d_{\alpha_{i} \beta_{i}}\left(1+\frac{1}{2} S_{\alpha_{i} \beta_{i}}\left(R_{i}\right) \cdot \frac{\partial}{\partial P_{i}}\right) \delta_{\alpha_{i}^{\prime} \beta_{i}^{\prime}}
\end{aligned}
$$

where $S_{\alpha_{i} \beta_{i}}=\left(E_{\alpha_{i}}-E_{\beta_{i}}\right) d_{\alpha_{i} \beta_{i}}\left(\frac{P}{M} \cdot d_{\alpha_{i} \beta_{i}}\right)^{-1}$ and $d_{\alpha_{i} \beta_{i}}=<\alpha_{i} ; R\left|\nabla_{R}\right| \beta_{i} ; R>$ is the non-adiabatic coupling matrix element.

The formal solution of Eq. (32) is

$$
W^{\alpha_{1}^{\prime} \alpha_{1} \alpha_{2}^{\prime} \alpha_{2}}(t)=\left(\exp \left(\frac{1}{2} \mathcal{K} t\right) \mathbf{W}(0)\right)_{\alpha_{1}^{\prime} \alpha_{1} \alpha_{2}^{\prime} \alpha_{2}} .
$$

Now that the quantum-classical evolution equation for the matrix elements of $W$ and its formal solution have been determined, we can return to the calculation of the quantum-classical limit of the quantum time correlation function.

\section{QUANTUM-CLASSICAL CORRELATION FUNCTION}

Equation (32) is one of the main results of this paper. Using it we can obtain a quantum-classical approximation to the quantum correlation function by replacing the full quantum evolution of the spectral density in Eq. (11) with 
its evolution in the quantum-classical limit given by Eq. (36), the solution of Eq. (32). We have

$$
\begin{aligned}
C_{A B}(t ; \beta)= & \frac{1}{\beta} \int_{0}^{\beta} d \lambda \sum_{\alpha_{1}, \alpha_{1}^{\prime}, \alpha_{2}, \alpha_{2}^{\prime}} \int \prod_{i=1}^{2} d X_{i} B_{W}^{\dagger \alpha_{1} \alpha_{1}^{\prime}}\left(X_{1}\right) A_{W}^{\alpha_{2} \alpha_{2}^{\prime}}\left(X_{2}\right) \\
& \times\left(\exp \left(\frac{1}{2} \mathcal{K} t\right) \mathbf{W}\left(X_{1}, X_{2}, 0 ; \beta, \lambda\right)\right)_{\alpha_{1}^{\prime} \alpha_{1} \alpha_{2}^{\prime} \alpha_{2}} .
\end{aligned}
$$

Since the operator $\mathcal{K}$ is the sum of two operators, one acting only on functions of $X_{1}$ and quantum indices with subscript 1, and the other on functions of $X_{2}$ and quantum indices with subscript 2, we may integrate by parts to have the operator act on the dynamical variables instead of $\mathbf{W}$. We obtain,

$$
C_{A B}(t, \beta)=\sum_{\beta_{1}^{\prime} \beta_{1} \beta_{2}^{\prime} \beta_{2}} \int \prod_{i=1}^{2} d X_{i} B_{W}^{\dagger \beta_{1} \beta_{1}^{\prime}}\left(X_{1}, \frac{t}{2}\right) A_{W}^{\beta_{2} \beta_{2}^{\prime}}\left(X_{2},-\frac{t}{2}\right) \bar{W}^{\beta_{1}^{\prime} \beta_{1} \beta_{2}^{\prime} \beta_{2}}\left(X_{1}, X_{2} ; \beta\right),
$$

where

$$
\begin{aligned}
B_{W}^{\dagger \beta_{1} \beta_{1}^{\prime}}\left(X_{1}, \frac{t}{2}\right) & =\sum_{\alpha_{1}^{\prime} \alpha_{1}}\left(e^{i \frac{t}{2} \mathcal{L}\left(X_{1}\right)}\right)_{\beta_{1} \beta_{1}^{\prime} \alpha_{1} \alpha_{1}^{\prime}} \hat{B}_{W}^{\dagger \alpha_{1} \alpha_{1}^{\prime}}\left(X_{1}\right), \\
A_{W}^{\beta_{2} \beta_{2}^{\prime}}\left(X_{2},-\frac{t}{2}\right) & =\sum_{\alpha_{2}^{\prime} \alpha_{2}}\left(e^{-i \frac{t}{2} \mathcal{L}\left(X_{2}\right)}\right)_{\beta_{2} \beta_{2}^{\prime} \alpha_{2} \alpha_{2}^{\prime}} \hat{A}_{W}^{\alpha_{2} \alpha_{2}^{\prime}}\left(X_{2}\right) .
\end{aligned}
$$

In writing Eq. (38) we defined

$$
\bar{W}^{\beta_{1}^{\prime} \beta_{1} \beta_{2}^{\prime} \beta_{2}}\left(X_{1}, X_{2} ; \beta\right)=\frac{1}{\beta} \int_{0}^{\beta} d \lambda W^{\beta_{1}^{\prime} \beta_{1} \beta_{2}^{\prime} \beta_{2}}\left(X_{1}, X_{2}, 0 ; \beta, \lambda\right)
$$

Equation (38) shows that the correlation function at time $t$ can be calculated by sampling $X_{1}$ and $X_{2}$ from suitable weights determined by $\bar{W}^{\beta_{1}^{\prime} \beta_{1} \beta_{2}^{\prime} \beta_{2}}\left(X_{1}, X_{2} ; \beta\right)$ at time zero and propagating $B_{W}^{\dagger \alpha_{1} \alpha_{1}^{\prime}}$ forward in time and $A_{W}^{\alpha_{2} \alpha_{2}^{\prime}}$ backward in time for an interval of length $t / 2$. Note that while the time evolution in Eq. (38) is by quantum-classical dynamics, the initial condition for $\bar{W}^{\beta_{1}^{\prime} \beta_{1} \beta_{2}^{\prime} \beta_{2}}\left(X_{1}, X_{2} ; \beta\right)$ is still an exact expression for the full equilibrium quantum mechanical spectral density.

\section{HIGH TEMPERATURE FORM OF $\overline{\mathrm{W}}$}

At $t=0 \mathbf{W}$ is given explicitly by

$$
\begin{aligned}
W^{\alpha_{1}^{\prime} \alpha_{1} \alpha_{2}^{\prime} \alpha_{2}}\left(X_{1}, X_{2}, 0 ; \beta, \lambda\right) & =\frac{1}{(2 \pi \hbar)^{2} \nu_{h}} Z_{Q} \int d Z_{1} d Z_{2} e^{-\frac{i}{\hbar}\left(P_{1} Z_{1}+P_{2} Z_{2}\right)} \\
& \times\left\langle\alpha_{1}^{\prime} ; R_{1}\left|\left\langle R_{1}+\frac{Z_{1}}{2}\left|e^{-(\beta-\lambda) \hat{H}}\right| R_{2}-\frac{Z_{2}}{2}\right\rangle\right| \alpha_{2} ; R_{2}\right\rangle \\
& \times\left\langle\alpha_{2}^{\prime} ; R_{2}\left|\left\langle R_{2}+\frac{Z_{2}}{2}\left|e^{-\lambda \hat{H}}\right| R_{1}-\frac{Z_{1}}{2}\right\rangle\right| \alpha_{1} ; R_{1}\right\rangle
\end{aligned}
$$


It can be computed using path integral techniques but its evaluation is still a difficult problem. In order to illustrate its structure we consider its form in the high temperature limit. In this limit we may write

$$
\left\langle R_{2}+\frac{Z_{2}}{2}\left|e^{-\lambda \hat{H}}\right| R_{1}-\frac{Z_{1}}{2}\right\rangle \approx e^{-\lambda \hat{h}\left(R_{c}-\frac{1}{4} Z_{12}\right)}\left(\frac{M}{2 \pi \lambda \hbar^{2}}\right)^{\nu_{h} / 2} \exp \left[-\frac{M\left(R_{12}-Z_{c}\right)^{2}}{2 \lambda \hbar^{2}}\right]
$$

where $\hat{h}=\frac{\hat{p}^{2}}{2 m}+\hat{V}$ and we have introduced the variables $Z_{c}=\left(Z_{1}+Z_{2}\right) / 2, Z_{12}=Z_{1}-Z_{2}, R_{c}=\left(R_{1}+R_{2}\right) / 2$ and $R_{12}=R_{1}-R_{2}$. Taking the desired matrix element of this expression and inserting complete sets of adiabatic states we obtain

$$
\begin{aligned}
\left\langle\alpha_{2}^{\prime} ; R_{2}\left|\left\langle R_{2}+\frac{Z_{2}}{2}\left|e^{-\lambda \hat{h}}\right| R_{1}-\frac{Z_{1}}{2}\right\rangle\right| \alpha_{1} ; R_{1}\right\rangle & =\sum_{\alpha} e^{-\lambda E_{\alpha}\left(R_{c}-\frac{Z_{12}}{2}\right)}\left\langle\alpha_{2}^{\prime} ; R_{2} \mid \alpha ; R_{c}-\frac{Z_{12}}{4}\right\rangle\left\langle\alpha ; R_{c}-\frac{Z_{12}}{4} \mid \alpha_{1} ; R_{1}\right\rangle \\
& =\sum_{\alpha} e^{-\lambda E_{\alpha}\left(R_{c}\right)}\left\langle\alpha_{2}^{\prime} ; R_{2} \mid \alpha ; R_{c}\right\rangle\left\langle\alpha ; R_{c} \mid \alpha_{1} ; R_{1}\right\rangle+\mathcal{O}\left(Z_{12}\right)
\end{aligned}
$$

Keeping only the zero order term in $Z_{12}$, the integral over $Z_{12}$ in Eq. (41) gives a factor $(4 \pi \hbar)^{\nu_{h}} \delta\left(P_{1}-P_{2}\right)$. The other term in Eq. (41), which arises from the combination of the gaussian on the right hand side of Eq. (42) along with the analogous expression coming from the high temperature limit of $\left\langle R_{1}+\frac{Z_{1}}{2}\left|e^{-(\beta-\lambda) \hat{H}}\right| R_{2}-\frac{Z_{2}}{2}\right\rangle$, can be evaluated by performing the gaussian integral on $Z_{c}$ to obtain

$$
\left(\frac{M}{2 \pi \hbar^{2} \beta}\right)^{\nu_{h} / 2} e^{\frac{i}{\hbar} 2 P_{c} \frac{2 \lambda-\beta}{\beta} R_{12}} e^{-\frac{2 M}{\hbar^{2}(\beta)} R_{12}^{2}} e^{-\frac{\lambda(\beta-\lambda)}{\beta} \frac{2 P_{c}^{2}}{M}}=\left(\frac{M}{2 \pi \hbar^{2} \beta}\right)^{\nu_{h} / 2} f\left(R_{12}, P_{c}\right) e^{-\frac{\lambda(\beta-\lambda)}{\beta} \frac{2 P_{c}^{2}}{M}}
$$

where $P_{c}=\left(P_{1}+P_{2}\right) / 2$. The function $f\left(R_{12}, P_{c}\right)$ still contains quantum information since it is composed of a phase factor and a gaussian expressing quantum dispersion effects in the heavy mass coordinates. We can obtain a classical bath approximation if we represent $f\left(R_{12}, P_{c}\right)$ in a multipole expansion and keep only the first order term, $f\left(R_{12}, P_{c}\right) \approx\left[\int d R_{12} f\left(R_{12}, P_{c}\right)\right] \delta\left(R_{12}\right)$, we have

$$
f\left(R_{12}, P_{c}\right) \approx\left(\frac{\pi \hbar^{2} \beta}{2 M}\right)^{\nu_{h} / 2} e^{-\frac{P_{c}^{2}}{2 \beta M}(2 \lambda-\beta)^{2}} \delta\left(R_{12}\right)
$$

Combining terms we obtain a high-temperature, classical-bath approximation to $\mathbf{W}$ :

$$
W^{\alpha_{1}^{\prime} \alpha_{1} \alpha_{2}^{\prime} \alpha_{2}}\left(X_{1}, X_{2}, 0 ; \beta, \lambda\right) \approx \frac{1}{(2 \pi \hbar)^{\nu_{h}} Z_{Q}} e^{-\beta \frac{P_{1}^{2}}{2 M}} e^{-(\beta-\lambda) E_{\alpha_{1}^{\prime}}\left(R_{1}\right)} e^{-\lambda E_{\alpha_{2}^{\prime}}\left(R_{1}\right)} \delta_{\alpha_{1}^{\prime} \alpha_{2}} \delta_{\alpha_{2}^{\prime} \alpha ; R_{1}} \delta\left(R_{12}\right) \delta\left(P_{12}\right)
$$

Thus the quantity $\overline{\mathbf{W}}$, defined in Eq. (40), is given in the high-temperature, classical-bath limit by

$$
\begin{aligned}
\bar{W}^{\alpha_{1}^{\prime} \alpha_{1} \alpha_{2}^{\prime} \alpha_{2}}\left(X_{1}, X_{2} ; \beta\right) & =\frac{1}{(2 \pi \hbar)^{\nu_{h}} Z_{Q}} e^{-\beta\left(\frac{P_{1}^{2}}{2 M}+E_{\alpha_{1}^{\prime}}\left(R_{1}\right)\right)} \frac{e^{\beta\left(E_{\alpha_{1}^{\prime}}\left(R_{1}\right)-E_{\alpha_{2}^{\prime}}\left(R_{1}\right)\right)}-1}{\beta\left(E_{\alpha_{1}^{\prime}}\left(R_{1}\right)-E_{\alpha_{2}^{\prime}}\left(R_{1}\right)\right)} \\
& \times \delta_{\alpha_{1}^{\prime} \alpha_{2}} \delta_{\alpha_{2}^{\prime} \alpha ; R_{1}} \delta\left(R_{12}\right) \delta\left(P_{12}\right) .
\end{aligned}
$$

Using similar manipulations, the high temperature limit of $Z_{Q}$ is

$$
Z_{Q} \approx \frac{1}{(2 \pi \hbar)^{\nu_{h}}} \sum_{\alpha} \int d R d P e^{-\beta\left(\frac{P^{2}}{2 M}+E_{\alpha}(R)\right)} .
$$


If Eq. (47) is used in the correlation function formula, Eq. (38), the result maybe shown to correspond with the quantum-classical linear response theory form [29] to lowest order in $\hbar$.

\section{CONCLUSION}

The expression for the quantum-classical limit of the quantum correlation function derived in this paper provides a route for the calculation of quantum transport properties in condensed phase systems. Difficult many-body quantum dynamics is replaced by quantum-classical evolution which can be carried out using surface-hopping schemes involving probabilistic sampling of quantum transitions, with associated momentum changes in the bath, and classical trajectory segments. The classical trajectory segments are accompanied by phase factors that account for quantum coherence

when off-diagonal matrix elements appear. 21] The full equilibrium quantum structure of the entire system is retained. While the equilibrium calculation is still a difficult problem it is more tractable than the quantum dynamics needed to treat the many-body system using full quantum dynamics. For example, imaginary time Feynman path integral methods for computing equilibrium properties are far more tractable than their corresponding real time variants. Since quantum information about the entire system is retained in the equilibrium structure, the formula for the correlation function incorporates some aspects of nuclear bath quantum dispersion that is missing in other quantum-classical schemes. The importance of retaining the full quantum equilibrium structure has been noted in Ref. [33].

The results also provide a framework for exploring and extending the statistical mechanics of quantum-classical systems. The correlation functions for transport properties that result from linear response theory in quantum-classical systems involve both quantum-classical evolution like that derived in this paper, as well as the equilibrium quantumclassical density that is stationary under the quantum-classical evolution. [29, 30] One may construct approximations to quantum transport properties by considering other approximate limiting forms of the equilibrium spectral density. We also note that to establish a complete comparison with quantum-classical linear response theory requires the retention of terms that were neglected in the calculations for $\overline{\mathbf{W}}$ presented in Sec. $\bar{\nabla}$ It should be fruitful to pursue extensions of such calculations to obtain other approximations for quantum transport properties.

\section{Acknowledgements}

This work was supported in part by a grant from the Natural Sciences and Engineering Research Council of Canada. RK would like to thank S. Bonella and G. Ciccotti for discussions pertaining to part of the work presented here. 


\section{Appendix A: DERIVATION OF QUANTUM-CLASSICAL EVOLUTION EQUATION FOR $W$}

The equation of motion for the spectral density (Eq. (21) takes a similar form in scaled coordinates:

$$
\begin{aligned}
\frac{\partial}{\partial t} W^{\prime}\left(x_{1}^{\prime}, x_{2}^{\prime}, X_{1}^{\prime}, X_{2}^{\prime}, t^{\prime} ; \beta^{\prime}, \lambda^{\prime}\right) & =-\frac{1}{2}\left(i L_{1}^{(0) \prime}\left(x_{1}^{\prime}, X_{1}^{\prime}\right)-i L_{2}^{(0) \prime}\left(x_{2}^{\prime}, X_{2}^{\prime}\right)\right) W^{\prime}\left(x_{1}^{\prime}, x_{2}^{\prime}, X_{1}^{\prime}, X_{2}^{\prime}, t^{\prime} ; \beta^{\prime}, \lambda^{\prime}\right) \\
& +\frac{1}{2} \int \prod_{i=1}^{2} d s_{i}^{\prime} d S_{i}^{\prime}\left(\omega_{1}^{\prime}\left(r_{1}^{\prime}, s_{1}^{\prime}, R_{1}^{\prime}, S_{1}^{\prime}\right) \delta\left(s_{2}^{\prime}\right) \delta\left(S_{2}^{\prime}\right)-\omega_{2}^{\prime}\left(r_{2}^{\prime}, s_{2}^{\prime}, R_{2}^{\prime}, S_{2}^{\prime}\right) \delta\left(s_{1}^{\prime}\right) \delta\left(S_{1}^{\prime}\right)\right) \\
& \times W^{\prime}\left(x_{1}^{\prime}-\pi_{1}^{\prime}, X_{1}^{\prime}-\Pi_{1}^{\prime}, x_{2}^{\prime}-\pi_{2}^{\prime}, X_{2}^{\prime}-\Pi_{2}^{\prime}, t^{\prime} ; \beta^{\prime}, \lambda^{\prime}\right),
\end{aligned}
$$

where the scaled free streaming Liouville operator and integral kernel are defined in Eqs. (24) and (25), respectively.

Inserting Eq. (26) into the expression for $\omega_{1}^{\prime}$ and retaining only terms up to linear order in in $\mu$ we find

$$
\begin{aligned}
\omega_{1}^{\prime}\left(r_{1}^{\prime}, s_{1}^{\prime}, R_{1}^{\prime}, S_{1}^{\prime}\right) \approx & \frac{2}{(\pi)^{\nu}} \int d \bar{r}_{1}^{\prime} d \tilde{R}_{1}^{\prime} V^{\prime}\left(r_{1}^{\prime}-\bar{r}_{1}^{\prime}, R_{1}^{\prime}\right) \sin \left(2 s_{1}^{\prime} \cdot \bar{r}_{1}^{\prime}+2 S_{1}^{\prime} \cdot \tilde{R}_{1}^{\prime}\right) \\
& -\frac{2 \mu}{(\pi)^{\nu}} \int d \bar{r}_{1}^{\prime} d \tilde{R}_{1}^{\prime} \frac{\partial V^{\prime}\left(r_{1}^{\prime}-\bar{r}_{1}^{\prime}, R_{1}^{\prime}\right)}{\partial R_{1}^{\prime}} \cdot \tilde{R}_{1}^{\prime} \sin \left(2 s_{1}^{\prime} \cdot \bar{r}_{1}^{\prime}+2 S_{1}^{\prime} \cdot \tilde{R}_{1}^{\prime}\right)+\mathcal{O}\left(\mu^{2}\right) .
\end{aligned}
$$

We observe that

$$
\begin{aligned}
\int d \bar{r}_{1}^{\prime} d \tilde{R}_{1}^{\prime} V^{\prime}\left(r_{1}^{\prime}-\bar{r}_{1}^{\prime}, R_{1}^{\prime}\right) \sin \left(2 s_{1}^{\prime} \cdot \bar{r}_{1}^{\prime}+2 S_{1}^{\prime} \cdot \tilde{R}_{1}^{\prime}\right)= & \int d \bar{r}_{1}^{\prime} d \tilde{R}_{1}^{\prime} V^{\prime}\left(r_{1}^{\prime}-\bar{r}_{1}^{\prime}, R_{1}^{\prime}\right)\left[\sin \left(2 s_{1}^{\prime} \cdot \bar{r}_{1}^{\prime}\right) \cos \left(2 S_{1}^{\prime} \cdot \tilde{R}_{1}^{\prime}\right)\right. \\
& \left.+\cos \left(2 s_{1}^{\prime} \cdot \bar{r}_{1}^{\prime}\right) \sin \left(2 S_{1}^{\prime} \cdot \tilde{R}_{1}^{\prime}\right)\right]
\end{aligned}
$$

using the trigonometric identity for the sine of a sum of arguments. Then, using the fact that $\int d \tilde{R}_{1}^{\prime} \cos \left(2 S_{1}^{\prime} \tilde{R}_{1}^{\prime}\right)=$ $\pi^{\nu_{\mathrm{h}}} \delta\left(S_{1}^{\prime}\right)$ we have

$$
\int d \bar{r}_{1}^{\prime} d \tilde{R}_{1}^{\prime} V^{\prime}\left(r_{1}^{\prime}-\bar{r}_{1}^{\prime}, R_{1}^{\prime}\right) \sin \left(2 s_{1}^{\prime} \cdot \bar{r}_{1}^{\prime}+2 S_{1}^{\prime} \cdot \tilde{R}_{1}^{\prime}\right)=\pi^{\nu_{\mathrm{h}}} \int d \bar{r}_{1}^{\prime} V^{\prime}\left(r_{1}^{\prime}-\bar{r}_{1}^{\prime}, R_{1}^{\prime}\right) \sin \left(2 s_{1}^{\prime} \cdot \bar{r}_{1}^{\prime}\right) \delta\left(S_{1}^{\prime}\right),
$$

where we have used $\int d \tilde{R}_{1}^{\prime} \sin \left(2 S_{1}^{\prime} \cdot \tilde{R}_{1}^{\prime}\right)=0$. In a similar manner

$$
\int d \bar{r}_{1}^{\prime} d \tilde{R}_{1}^{\prime} \frac{\partial V^{\prime}\left(r_{1}^{\prime}-\bar{r}_{1}^{\prime}, R_{1}^{\prime}\right)}{\partial R_{1}^{\prime}} \cdot \tilde{R}_{1}^{\prime} \sin \left(2 s_{1}^{\prime} \cdot \bar{r}_{1}^{\prime}+2 S_{1}^{\prime} \cdot \tilde{R}_{1}^{\prime}\right)=-\frac{\pi^{\nu_{\mathrm{h}}}}{2} \int d \bar{r}_{1}^{\prime} \frac{\partial V^{\prime}\left(r_{1}^{\prime}-\bar{r}_{1}^{\prime}, R_{1}^{\prime}\right)}{\partial R_{1}^{\prime}} \cos \left(2 s_{1}^{\prime} \cdot \bar{r}_{1}^{\prime}\right) \cdot \frac{d \delta\left(S_{1}^{\prime}\right)}{d S_{1}^{\prime}}
$$

where we have used the relations $\int d \tilde{R}_{1}^{\prime} \tilde{R}_{1}^{\prime} \cos \left(2 S_{1}^{\prime} \cdot \tilde{R}_{1}^{\prime}\right)=0$ and $\int d \tilde{R}_{1}^{\prime} \tilde{R}_{1}^{\prime} \sin \left(2 S_{1}^{\prime} \cdot \tilde{R}_{1}^{\prime}\right)=-\left(\pi^{\nu_{\mathrm{h}}} / 2\right) d \delta\left(S_{1}^{\prime}\right) / d S_{1}^{\prime}$. Then to order $\mathcal{O}(\mu)$,

$$
\begin{aligned}
\omega_{1}^{\prime}\left(r_{1}^{\prime}, s_{1}^{\prime}, R_{1}^{\prime}, S_{1}^{\prime}\right) & =\frac{2}{\pi^{\nu \ell}} \int d \bar{q}^{\prime} V^{\prime}\left(r_{1}^{\prime}-\bar{r}_{1}^{\prime}, R_{1}^{\prime}\right) \sin \left(2 s_{1}^{\prime} \cdot \bar{r}_{1}^{\prime}\right) \delta\left(S_{1}^{\prime}\right) \\
& +\mu\left[\frac{1}{\pi^{\nu \ell}} \int d \bar{r}_{1}^{\prime} \frac{\partial V^{\prime}\left(r_{1}^{\prime}-\bar{r}_{1}^{\prime}, R_{1}^{\prime}\right)}{\partial R_{1}^{\prime}} \cos \left(2 s_{1}^{\prime} \cdot \bar{r}_{1}^{\prime}\right) \cdot \frac{d \delta\left(S_{1}^{\prime}\right)}{d S_{1}^{\prime}}\right]
\end{aligned}
$$

Using this expression we may compute the integral on the right hand side of Eq. A1 involving $\omega_{1}^{\prime}$. The algebra for the $\omega_{2}^{\prime}$ term is similar. Given the expression (A6), the integral

$$
\int d s_{1}^{\prime} d S_{1}^{\prime} \omega_{1}^{\prime}\left(r_{1}^{\prime}, s_{1}^{\prime}, R_{1}^{\prime}, S_{1}^{\prime}\right) W^{\prime}\left(x_{1}^{\prime}-\pi_{1}^{\prime}, X_{1}^{\prime}-\Pi_{1}^{\prime}, x_{2}^{\prime}, X_{2}^{\prime}, t^{\prime} ; \beta, \lambda\right)
$$


has two contributions. The first is is

$$
\begin{gathered}
\frac{2}{\pi^{\nu_{\ell}}} \int d s_{1}^{\prime} d S_{1}^{\prime} W^{\prime}\left(x_{1}^{\prime}-\pi_{1}^{\prime}, X_{1}^{\prime}-\Pi_{1}^{\prime}, x_{2}^{\prime}, X_{2}^{\prime}, t^{\prime} ; \beta, \lambda\right) \int d \bar{r}_{1}^{\prime} V^{\prime}\left(r_{1}^{\prime}-\bar{r}_{1}^{\prime}, R_{1}^{\prime}\right) \sin \left(2 s_{1}^{\prime} \cdot \bar{r}_{1}^{\prime}\right) \delta\left(S_{1}^{\prime}\right)= \\
\frac{2}{\pi^{\nu_{\ell}}} \int d s_{1}^{\prime} W^{\prime}\left(x_{1}^{\prime}-\pi_{1}^{\prime}, X_{1}^{\prime}, x_{2}^{\prime}, X_{2}^{\prime} ; t^{\prime}, \beta, \lambda\right) \int d \bar{r}_{1}^{\prime} V^{\prime}\left(r_{1}^{\prime}-\bar{r}_{1}^{\prime}, R_{1}^{\prime}\right) \sin \left(2 s_{1}^{\prime} \cdot \bar{r}_{1}^{\prime}\right),
\end{gathered}
$$

while the second is

$$
\begin{gathered}
\frac{\mu}{\pi^{\nu_{\ell}}} \int d s_{1}^{\prime} d S_{1}^{\prime} W^{\prime}\left(x_{1}-\pi_{1}^{\prime}, X_{1}^{\prime}-\Pi_{1}^{\prime}, x_{2}^{\prime}, X_{2}^{\prime}, t^{\prime} ; \beta, \lambda\right) \int d \bar{r}_{1}^{\prime} \frac{\partial}{\partial R_{1}^{\prime}} V^{\prime}\left(r_{1}^{\prime}-\bar{r}_{1}^{\prime}, R_{1}^{\prime}\right) \cos \left(2 s_{1}^{\prime} \cdot \bar{r}_{1}^{\prime}\right) \cdot \frac{d \delta\left(S_{1}^{\prime}\right)}{d S_{1}^{\prime}}= \\
\frac{\mu}{\pi^{\nu_{\ell}}} \int d s_{1}^{\prime} \frac{\partial}{\partial P_{1}^{\prime}} W^{\prime}\left(x_{1}^{\prime}-\pi_{1}^{\prime}, X_{1}^{\prime}, x_{2}^{\prime}, X_{2}^{\prime}, t^{\prime} ; \beta, \lambda\right) \cdot \int d \bar{r}_{1}^{\prime} \frac{\partial}{\partial R_{1}^{\prime}} V^{\prime}\left(r_{1}^{\prime}-\bar{r}_{1}^{\prime}, R_{1}^{\prime}\right) \cos \left(2 s_{1}^{\prime} \cdot \bar{r}_{1}^{\prime}\right) .
\end{gathered}
$$

Defining $\Delta F_{R_{1}^{\prime}, s_{1}^{\prime}}^{\prime}=-\frac{\partial}{\partial R_{1}^{\prime}}\left[V^{\prime}\left(R_{1}^{\prime}\right) \delta\left(s_{1}^{\prime}\right)-\frac{1}{\pi^{\nu \ell}} \int d \bar{r}_{1}^{\prime} \cos \left(2 s_{1}^{\prime} \cdot \bar{r}_{1}^{\prime}\right) V^{\prime}\left(r_{1}^{\prime}-\bar{r}_{1}^{\prime}, R_{1}^{\prime}\right)\right]$ and returning to unscaled coordinates we obtain Eq. (27), the desired quantum-classical evolution equation for the full Wigner representation of $W$. The first term in the definition of $\Delta F^{\prime}$ is compensated by the introduction of the full classical propagator for the heavy mass degrees of freedom. Written in this form, $\Delta F^{\prime}$ also depends only on the interaction potential between the light and heavy mass particles.

\section{Appendix B: EQUATION IN THE PARTIAL WIGNER REPRESENTATION}

In this appendix we perform explicitly the calculations that, starting from Eq. (27), lead to Eq. (32). This calculation

amounts to the evaluation of $\left(\mathcal{T}^{-1} \circ K \circ \mathcal{T}\right) \circ \mathbf{W}(t)$. The various terms in $K$, defined by Eq. (27), are considered separately.

Consider the calculation of $\left(\mathcal{T}^{-1} \circ i L_{1}\left(X_{1}\right) \circ \mathcal{T}\right) \circ \mathbf{W}$ which is composed of two terms. The force term is $\mathcal{T}^{-1} \circ$ $F_{R_{1}} \cdot \partial / \partial P_{1} \mathcal{T} \circ \mathbf{W}=F_{R_{1}} \cdot \partial / \partial P_{1} \mathbf{W}$, since $\mathcal{T}$ and its inverse do not depend on $P_{1}$. The free streaming term $\left(\mathcal{T}^{-1} \circ \frac{P_{1}}{M} \cdot \frac{\partial}{\partial R_{1}} \circ \mathcal{T}\right) \circ \mathbf{W}$ requires additional calculations since $\mathcal{T}$ depends on $R_{1}$. We have

$$
\begin{aligned}
\left(\mathcal{T}^{-1} \circ \frac{P_{1}}{M} \cdot \frac{\partial}{\partial R_{1}}\right. & \circ \mathcal{T}) \circ \mathbf{W}=\frac{P_{1}}{M} \cdot \sum_{\beta_{1} \beta_{1}^{\prime} \beta_{2} \beta_{2}^{\prime}} \int \prod_{j=1}^{2} d r_{j} d z_{j} \phi_{\alpha_{1}^{\prime}}^{*}\left(r_{1}+\frac{z_{1}}{2}, R_{1}\right) \phi_{\alpha_{1}}\left(r_{1}-\frac{z_{1}}{2}, R_{1}\right) \\
& \times \phi_{\alpha_{2}^{\prime}}^{*}\left(q_{2}+\frac{z_{2}}{2} ; R_{2}\right) \phi_{\alpha_{2}}\left(r_{2}-\frac{z_{2}}{2} ; R_{2}\right) \phi_{\beta_{2}^{\prime}}\left(r_{2}+\frac{z_{2}}{2} ; R_{2}\right) \phi_{\beta_{2}}^{*}\left(q_{2}-\frac{z_{2}}{2} ; R_{2}\right) \\
& \times\left\{\left[\frac{\partial \phi_{\beta_{1}^{\prime}}\left(r_{1}+\frac{z_{1}}{2} ; R_{1}\right)}{\partial R_{1}} \phi_{\beta_{1}}^{*}\left(r_{1}-\frac{z_{1}}{2} ; R_{1}\right)+\phi_{\beta_{1}^{\prime}}\left(r_{1}+\frac{z_{1}}{2} ; R_{1}\right) \frac{\partial \phi_{\beta_{1}}^{*}\left(r_{1}-\frac{z_{1}}{2} ; R_{1}\right)}{\partial R_{1}}\right] W^{\beta_{1}^{\prime} \beta_{1} \beta_{2}^{\prime} \beta_{2}}\right. \\
& \left.+\phi_{\beta_{1}^{\prime}}\left(r_{1}+\frac{z_{1}}{2} ; R_{1}\right) \phi_{\beta_{1}}^{*}\left(r_{1}-\frac{z_{1}}{2} ; R_{1}\right) \frac{\partial}{\partial R_{1}} W^{\beta_{1}^{\prime} \beta_{1} \beta_{2}^{\prime} \beta_{2}}\right\} .
\end{aligned}
$$

The last term where $\partial \mathbf{W} / \partial R_{1}$ appears is simple to calculate and gives $\frac{P_{1}}{M} \cdot \frac{\partial}{\partial R_{1}} W^{\alpha_{1}^{\prime} \alpha_{1} \alpha_{2}^{\prime} \alpha_{2}}$. To calculate the other two terms, we make the change of variables $q_{1}=r_{1}-\frac{z_{1}}{2}, q_{2}=r_{1}+\frac{z_{1}}{2}, q_{3}=r_{2}-\frac{z_{2}}{2}, q_{4}=r_{2}+\frac{z_{2}}{2}$. Integrating over 
$q_{3}$ and $q_{4}$ and using $\int d q \phi_{\alpha}(q, R) \phi_{\beta}^{*}(q, R)=\delta_{\alpha \beta}$, we obtain

$$
\begin{aligned}
& \frac{P_{1}}{M} \cdot \sum_{\beta_{1} \beta_{1}^{\prime}} \int d q_{1} d q_{2} \phi_{\alpha_{1}^{\prime}}^{*}\left(q_{2} ; R_{1}\right) \phi_{\alpha_{1}}\left(q_{1} ; R_{1}\right)\left[\frac{\partial \phi_{\beta_{1}^{\prime}}\left(q_{2} ; R_{1}\right)}{\partial R_{1}} \phi_{\beta_{1}}^{*}\left(q_{1} ; R_{1}\right)+\phi_{\beta_{1}^{\prime}}\left(q_{2} ; R_{1}\right) \frac{\partial \phi_{\beta_{1}}^{*}\left(q_{1} ; R_{1}\right)}{\partial R_{1}}\right] W^{\beta_{1}^{\prime} \beta_{1} \alpha_{2}^{\prime} \alpha_{2}} \\
& =\frac{P_{1}}{M} \cdot \sum_{\beta_{1}^{\prime}} d_{\alpha_{1}^{\prime} \beta_{1}^{\prime}} W^{\beta_{1}^{\prime} \alpha_{1} \alpha_{2}^{\prime} \alpha_{2}}-\frac{P_{1}}{M} \cdot \sum_{\beta_{1}} d_{\beta_{1} \alpha_{1}} W^{\alpha_{1}^{\prime} \beta_{1} \alpha_{2}^{\prime} \alpha_{2}}
\end{aligned}
$$

where we have introduced the definition of the nonadiabatic coupling vector.

Consider the calculation of $\left(\mathcal{T}^{-1} \circ i L_{1}^{(0)}\left(x_{1}\right) \circ \mathcal{T}\right) \circ \mathbf{W}$. In this case one integration over the momentum, arising from the definition of $\mathcal{T}^{-1}$, gives $\int d p_{1}\left(p_{1} / m\right) e^{\frac{i}{\hbar} p_{1}\left(z_{1}-z_{3}\right)}=(2 \pi \hbar)^{\nu_{\ell}}(i \hbar / m) \partial \delta\left(z_{1}-z_{3}\right) / \partial z_{3}$, while integration over $p_{2}$ gives $(2 \pi \hbar)^{\nu_{\ell}} \delta\left(z_{2}-z_{4}\right)$. One can then integrate by parts on $z_{3}$ and obtain

$$
\begin{aligned}
\left(\mathcal{T}^{-1} \circ \frac{p_{1}}{m} \quad\right. & \left.\frac{\partial}{\partial r_{1}} \circ \mathcal{T}\right) \circ \mathbf{W}=-\frac{i \hbar}{m} \sum_{\beta_{1} \beta_{1}^{\prime} \beta_{2} \beta_{2}^{\prime}} \int \prod_{j=1}^{2} d r_{j} \prod_{k=1}^{3} d z_{k} \delta\left(z_{1}-z_{3}\right) \phi_{\alpha_{1}^{\prime}}^{*}\left(r_{1}+\frac{z_{1}}{2} ; R_{1}\right) \phi_{\alpha_{1}}\left(r_{1}-\frac{z_{1}}{2} ; R_{1}\right) \\
& \times \phi_{\alpha_{2}^{\prime}}^{*}\left(r_{2}+\frac{z_{2}}{2} ; R_{2}\right) \phi_{\alpha_{2}}\left(r_{2}-\frac{z_{2}}{2} ; R_{2}\right) \phi_{\beta_{2}^{\prime}}\left(r_{2}+\frac{z_{2}}{2} ; R_{2}\right) \phi_{\beta_{2}}^{*}\left(r_{2}-\frac{z_{2}}{2} ; R_{2}\right) \\
& \times \frac{\partial^{2}}{\partial z_{3} \partial r_{1}}\left[\phi_{\beta_{1}^{\prime}}\left(r_{1}+\frac{z_{3}}{2} ; R_{1}\right) \phi_{\beta_{1}}^{*}\left(r_{1}-\frac{z_{3}}{2} ; R_{1}\right)\right] W^{\beta_{1}^{\prime} \beta_{1} \beta_{2}^{\prime} \beta_{2}} .
\end{aligned}
$$

Then use

$$
\begin{aligned}
\frac{\partial^{2}}{\partial z_{3} \partial r_{1}} \quad\left[\phi_{\beta_{1}^{\prime}}\left(r_{1}+\frac{z_{3}}{2} ; R_{1}\right) \phi_{\beta_{1}}^{*}\left(r_{1}-\frac{z_{3}}{2} ; R_{1}\right)\right] \\
\quad=\frac{1}{2}\left[\frac{\partial^{2} \phi_{\beta_{1}^{\prime}}\left(r_{1}+\frac{z_{3}}{2} ; R_{1}\right)}{\partial\left(r_{1}+\frac{z_{3}}{2}\right)^{2}} \phi_{\beta_{1}}^{*}\left(r_{1}-\frac{z_{3}}{2} ; R_{1}\right)-\phi_{\beta_{1}^{\prime}}\left(r_{1}+\frac{z_{3}}{2} ; R_{1}\right) \frac{\partial^{2} \phi_{\beta_{1}}^{*}\left(r_{1}-\frac{z_{3}}{2} ; R_{1}\right)}{\partial\left(r_{1}-\frac{z_{3}}{2}\right)^{2}}\right],
\end{aligned}
$$

go back to the integral, perform the integration on $z_{3}$ and make the change of variables previously introduced. The integration over $q_{3}$ and $q_{4}$ can be performed using the completeness of the adiabatic basis and one gets

$$
\begin{gathered}
\left(\mathcal{T}^{-1} \circ \frac{p_{1}}{m} \quad \frac{\partial}{\partial r_{1}} \circ \mathcal{T}\right) \circ \mathbf{W}=\frac{i}{\hbar} \sum_{\beta_{1}^{\prime}}\left\langle\alpha_{1}^{\prime} ; R_{1}\left|\frac{\hat{p}^{2}}{2 m}\right| \beta_{1}^{\prime} ; R_{1}\right\rangle W^{\beta_{1}^{\prime} \alpha_{1} \alpha_{2}^{\prime} \alpha_{2}} \\
-\frac{i}{\hbar} \sum_{\beta_{1}}\left\langle\beta_{1} ; R_{1}\left|\frac{\hat{p}^{2}}{2 m}\right| \alpha_{1} ; R_{1}\right\rangle W^{\alpha_{1}^{\prime} \beta_{1} \alpha_{2}^{\prime} \alpha_{2}}
\end{gathered}
$$

where we have used the identity $\left\langle\alpha, R\left|\hat{p}^{2} / 2 m\right| \beta, R\right\rangle=-\left(\hbar^{2} / 2 m\right) \int d q \phi_{\alpha}^{*}(q, R) \partial \phi_{\beta}(q, R) / \partial q^{2}$.

We consider now the transformation of the potential term in $K \circ W$ equal to $c_{1} \int d s_{1} d s_{2} \delta\left(s_{2}\right) \int d \bar{r} V\left(r_{1}-\right.$ $\bar{r}) \sin \left(\frac{2 s_{1} \cdot \bar{r}}{\hbar}\right) W\left(x_{1}-\pi_{1}, x_{2}-\pi_{2}, X_{1}, X_{2}, t ; \beta \lambda\right)$, where $c_{1}=2 \hbar^{-1}(\pi \hbar)^{-\nu_{\ell}}$; we may immediately perform the trivial integrations on $p_{1}, p_{2}, z_{3}$ and $z_{4}$. We also make the change variables below Eq. (B1) so that the integrations on $q_{3}$ and $q_{4}$ are also trivial. One is left with the integral

$$
\begin{aligned}
& c_{1} \sum_{\beta_{1}^{\prime} \beta_{1}} \int d q_{1} d q_{2} \int d s_{1} d \bar{r} V\left(\frac{q_{1}+q_{2}}{2}-\bar{r}\right) \sin \left(\frac{2 s_{1} \cdot \bar{r}}{\hbar}\right) e^{\frac{i}{\hbar} s_{1} \cdot\left(q_{2}-q_{1}\right)} \\
& \times \phi_{\alpha_{1}^{\prime}}^{*}\left(q_{2} ; R_{1}\right) \phi_{\alpha_{1}}\left(q_{1} ; R_{1}\right) \phi_{\beta_{1}^{\prime}}\left(q_{2} ; R_{1}\right) \phi_{\beta_{1}}^{*}\left(q_{1} ; R_{1}\right) W^{\beta_{1}^{\prime} \beta_{1} \alpha_{2}^{\prime} \alpha_{2}}
\end{aligned}
$$


Using the fact that $\int d s_{1} e^{\frac{i}{\hbar} s_{1} \cdot\left(q_{2}-q_{1}\right)} \sin \left(2 s_{1} \cdot \bar{r} / \hbar\right)=(2 \pi \hbar)^{\nu_{\ell}}\left[\delta\left(q_{2}-q_{1}+2 \bar{r}\right)-\delta\left(q_{2}-q_{1}-2 \bar{r}\right)\right] / 2 i$, substituting into Eq. (B-B) and making the change of variable $\sigma=2 \bar{r}$, the delta functions can be integrated out and one obtains,

$$
\begin{aligned}
& \frac{1}{i \hbar} \sum_{\beta_{1}^{\prime} \beta_{1}}\left[\int d q_{1} \phi_{\alpha_{1}}\left(q_{1} ; R_{1}\right) \phi_{\beta_{1}}^{*}\left(q_{1} ; R_{1}\right)\right]\left[\int d q_{2} \phi_{\alpha_{1}^{\prime}}^{*}\left(q_{2} ; R_{1}\right) V\left(q_{2}\right) \phi_{\beta_{1}^{\prime}}\left(q_{2} ; R_{1}\right)\right] W^{\beta_{1}^{\prime} \beta_{1} \alpha_{2}^{\prime} \alpha_{2}} \\
& -\frac{1}{i \hbar} \sum_{\beta_{1}^{\prime} \beta_{1}}\left[\int d q_{1} \phi_{\beta_{1}}^{*}\left(q_{1} ; R_{1}\right) V\left(q_{1}\right) \phi_{\alpha_{1}}\left(q_{1} ; R_{1}\right)\right]\left[\int d q_{2} \phi_{\alpha_{1}^{\prime}}^{*}\left(q_{2} ; R_{1}\right) \phi_{\beta_{1}^{\prime}}\left(q_{2} ; R_{1}\right)\right] W^{\beta_{1}^{\prime} \beta_{1} \alpha_{2}^{\prime} \alpha_{2}} \\
& =\frac{1}{i \hbar} \sum_{\beta_{1}^{\prime}}\left\langle\alpha_{1}^{\prime} ; R_{1}|\hat{V}| \beta_{1}^{\prime} ; R_{1}\right\rangle W^{\beta_{1}^{\prime} \alpha_{1} \alpha_{2}^{\prime} \alpha_{2}}-\frac{1}{i \hbar} \sum_{\beta_{1}}\left\langle\beta_{1} ; R_{1}|\hat{V}| \alpha_{1} ; R_{1}\right\rangle W^{\alpha_{1}^{\prime} \beta_{1} \alpha_{2}^{\prime} \alpha_{2}} .
\end{aligned}
$$

The last term that must be worked out explicitly arises from the transformation of the quantum-classical term $\int d s_{1} d s_{2} \delta\left(s_{2}\right) \Delta F_{1}\left(R_{1}, s_{1}\right) \cdot \frac{\partial}{\partial P_{1}} W\left(x_{1}-\pi_{1}, x_{2}-\pi_{2}, X_{1}, X_{2}, t ; \lambda, \beta\right)$. Recalling the expression for $\Delta F_{1}$ in Eq. (29) one sees that there are two contributions to transform. The transformation of the term involving $\frac{\partial V\left(R_{1}\right)}{\partial R_{1}} \delta\left(s_{1}\right)$ is the same as that for the force term in $i L_{1}\left(X_{1}\right)$ and yields $F_{R_{1}} \cdot \partial W^{\alpha_{1}^{\prime} \alpha_{1} \alpha_{2}^{\prime} \alpha_{2}} / \partial P_{1}$. The integral term in $\Delta F_{1}\left(R_{1}, s_{1}\right)$ can be computed by integrating over $p_{1}, p_{2}, z_{3}$ and $z_{4}$, performing the change of variables below Eq. (B1) and integrating over $q_{3}$ and $q_{4}$, to obtain

$$
\begin{aligned}
& \sum_{\beta_{1}^{\prime} \beta_{1}} \frac{1}{(\pi \hbar)^{\nu_{\ell}}} \int d q_{1} d q_{2} \phi_{\alpha_{1}^{\prime}}^{*}\left(q_{2} ; R_{1}\right) \phi_{\alpha_{1}}\left(q_{1} ; R_{1}\right) \phi_{\beta_{1}^{\prime}}\left(q_{2} ; R_{1}\right) \phi_{\beta_{1}}^{*}\left(q_{1} ; R_{1}\right) \\
& \times \int d s_{1} \int d \bar{r}\left(\frac{\partial}{\partial R_{1}} V\left(\frac{q_{1}+q_{2}}{2}-\bar{r}, R_{1}\right)\right) \cos \left(\frac{2 s_{1} \cdot \bar{r}}{\hbar}\right) e^{\frac{i}{\hbar} s_{1} \cdot\left(q_{2}-q_{1}\right)} \frac{\partial}{\partial P_{1}} W^{\beta_{1}^{\prime} \beta_{1} \alpha_{2}^{\prime} \alpha_{2}}
\end{aligned}
$$

Then one can use the integral $\int d s_{1} \cos \left(2 s_{1} \cdot \bar{r} / \hbar\right) e^{\frac{i}{\hbar} s_{1} \cdot\left(q_{2}-q_{1}\right)}=(2 \pi \hbar)^{\nu_{\ell}}\left[\delta\left(q_{2}-q_{1}+2 \bar{r}\right)+\delta\left(q_{2}-q_{1}-2 \bar{r}\right)\right] / 2$, make the change of variable $\sigma=2 \bar{r}$, and integrate out the delta functions to find

$$
\frac{1}{2}\left[\sum_{\beta_{1}^{\prime}}\left\langle\alpha_{1}^{\prime} ; R_{1}\left|\frac{\partial \hat{V}\left(R_{1}\right)}{\partial R_{1}}\right| \beta_{1}^{\prime} ; R_{1}\right\rangle \frac{\partial}{\partial P_{1}} W^{\beta_{1}^{\prime} \alpha_{1} \alpha_{2}^{\prime} \alpha_{2}}+\sum_{\beta_{1}}\left\langle\beta_{1} ; R_{1}\left|\frac{\partial \hat{V}\left(R_{1}\right)}{\partial R_{1}}\right| \alpha_{1} ; R_{1}\right\rangle \frac{\partial}{\partial P_{1}} W^{\alpha_{1}^{\prime} \beta_{1} \alpha_{2}^{\prime} \alpha_{2}}\right] .
$$

Analogous terms (but with opposite sign) are obtained when considering the transformation of the terms depending on $x_{2}$ and $X_{2}$ in Eq. (27).

Combining all terms and using the relations $F_{W}^{\alpha \beta}(R)=-\left\langle\alpha ; R\left|\nabla_{R} \hat{V}(R)\right| \beta ; R\right\rangle=F_{W}^{\alpha}+\left(E_{\alpha}-E_{\beta}\right) d_{\alpha \beta}$,

$$
\begin{aligned}
-\frac{i}{\hbar} \sum_{\beta_{1}^{\prime}}\left\langle\alpha_{1}^{\prime} ; R_{1}\left|\left(\frac{\hat{p}^{2}}{2 m}+\hat{V}\right)\right| \beta_{1}^{\prime} ; R_{1}\right\rangle W^{\beta_{1}^{\prime} \alpha_{1} \alpha_{2}^{\prime} \alpha_{2}} & +\frac{i}{\hbar} \sum_{\beta_{1}}\left\langle\beta_{1} ; R_{1}\left|\left(\frac{\hat{p}^{2}}{2 m}+\hat{V}\right)\right| \alpha_{1} ; R_{1}\right\rangle W^{\alpha_{1}^{\prime} \beta_{1} \alpha_{2}^{\prime} \alpha_{2}}= \\
=-\frac{i}{\hbar}\left(E_{\alpha_{1}^{\prime}}\left(R_{1}\right)-E_{\alpha_{1}}\left(R_{1}\right)\right) W^{\alpha_{1}^{\prime} \alpha_{1} \alpha_{2}^{\prime} \alpha_{2}} & =-i \omega_{\alpha_{1}^{\prime} \alpha_{1}}\left(R_{1}\right) W^{\alpha_{1}^{\prime} \alpha_{1} \alpha_{2}^{\prime} \alpha_{2}}
\end{aligned}
$$

and introducing the definition $S_{\alpha_{i} \beta_{i}}=\left(E_{\alpha_{i}}-E_{\beta_{i}}\right) d_{\alpha_{i} \beta_{i}}\left(\frac{P}{M} \cdot d_{\alpha_{i} \beta_{i}}\right)^{-1}$, we find Eq. (32).

[1] R. Kubo: J. Phys. Soc. (Japan) 12, 570 (1957); R. Kubo: Repts. Prog. Phys. 29, 255 (1966). 
[2] H. Mori, Prog. Theor. Phys. 33, 423 (1965).

[3] E. Rabani and D. Reichman, J. Chem. Phys. 120, 1458 (2004).

[4] S. A. Egorov, E. Rabani and B. J. Berne, J. Phys. Chem. B 103, 10978 (1999).

[5] A. A. Golosov, D. R. Reichman and E. Rabani, J. Chem. Phys. 118, 457 (2003).

[6] P. Pechukas, Phys. Rev. 181, 166, 174 (1969).

[7] M. F. Herman, Annu. Rev. Phys. Chem. 45, 83 (1994).

[8] J. C. Tully, Modern Methods for Multidimensional Dynamics Computations in Chemistry, ed. D. L. Thompson, (World Scientific, NY, 1998), p. 34 .

[9] J.C. Tully, J. Chem. Phys. 93, 1061 (1990); J.C. Tully: Int. J. Quantum Chem. 25, 299 (1991); S. Hammes-Schiffer and J.C. Tully: J. Chem. Phys. 101, 4657 (1994); D. S. Sholl and J. C. Tully, J. Chem. Phys. 109, 7702 (1998).

[10] L. Xiao and D. F. Coker, J. Chem. Phys. 100, 8646 (1994); D. F. Coker and L. Xiao: J. Chem. Phys. 102, 496 (1995); H. S. Mei and D.F. Coker: J. Chem. Phys. 104, 4755 (1996).

[11] F. Webster, P. J. Rossky, and P. A. Friesner, Comp. Phys. Comm. 63, 494 (1991); F. Webster, E. T. Wang, P. J. Rossky, and P. A. Friesner: J. Chem. Phys. 100, 483 (1994).

[12] T. J. Martinez, M. Ben-Nun, and R. D. Levine, J. Phys. Chem. A 101, 6389 (1997).

[13] A. Warshel and Z. T. Chu, J. Chem. Phys. 934003 (1990).

[14] I. V. Aleksandrov, Z. Naturforsch. 36a, 902 (1981).

[15] V. I. Gerasimenko, Repts. Ukranian Acad. Sci. 10, 65 (1981); Teo. Mat. Fiz. 150, 7 (1982).

[16] W. Boucher and J. Traschen, Phys. Rev. D 37, 3522 (1988).

[17] W. Y. Zhang and R. Balescu, J. Plasma Phys. 40, 199 (1988); R. Balescu and W. Y. Zhang, J. Plasma Phys. 40, 215 (1988).

[18] C. C. Martens and J.-Y. Fang, J. Chem. Phys. 106, 4918 (1996); A. Donoso and C. C. Martens, J. Phys. Chem. 102, 4291 (1998); D. Kohen and C. C. Martens, J. Chem. Phys. 111, 4343 (1999); 112, 7345 (2000).

[19] I. Horenko, C. Salzmann, B. Schmidt and C. Schütte, J. Chem. Phys. 117, 11075 (2002).

[20] C. Wan and J. Schofield: J. Chem. Phys. 113, 7047 (2000).

[21] R. Kapral and G. Ciccotti, J. Chem. Phys. 110, 8919 (1999).

[22] E. P. Wigner, Phys. Rev. 40, 749 (1932); K. Imre, E. Özizmir, M. Rosenbaum and P. F. Zwiefel, J. Math. Phys. 5, 1097 (1967); M. Hillery, R. F. O’Connell, M. O. Scully and E. P. Wigner, Phys. Repts. 106, 121 (1984).

[23] K. Thompson and N. Makri, J. Chem. Phys. 110, 1343 (1999).

[24] D. Mac Kernan, G. Ciccotti and R. Kapral, J. Chem. Phys. 106, (2002).

[25] D. Mac Kernan, G. Ciccotti and R. Kapral, J. Phys. Condens. Matt. 149069 (2002).

[26] S. Nielsen, R. Kapral and G. Ciccotti, J. Chem. Phys. 112, 6543 (2000). 
[27] S. Nielsen, R. Kapral and G. Ciccotti, J. Stat. Phys. 101, 225 (2000).

[28] A. Sergi, D. Mac Kernan, G. Ciccotti and R. Kapral, Theor. Chem. Acc. 11049 (2003).

[29] S. Nielsen, R. Kapral and G. Ciccotti, J. Chem. Phys. 115, 5805 (2001).

[30] R. Kapral and G. Ciccotti, A Statistical Mechanical Theory of Quantum Dynamics in Classical Environments, in Bridging Time Scales: Molecular Simulations for the Next Decade, 2001, eds. P. Nielaba, M. Mareschal and G. Ciccotti, (Springer, Berlin, 2003), p. 445.

[31] A. Sergi and R. Kapral, J. Chem. Phys., 118, 8566 (2003); J. Chem. Phys. 11912776 (2003).

[32] V. S. Filinov, Y. V. Medvedev and V. L. Kamskyi, Mol. Phys. 85711 (1995); V. S. Filinov, Mol. Phys. 88 1517 (1996); V. S. Filinov, Mol. Phys. 881529 (1996); V. S. Filinov, S. Bonella, Y. E. Lozovik, A. Filinov and I. Zacharov in Classical and Quantum Dynamics in Condensed Phase Simulations, edited by B. J. Berne, G. Ciccotti and D. F. Coker (World Scientific, Singapore, 1998), p. 667.

[33] S. A. Egorov, E. Rabani and B. J. Berne, J. Chem. Phys. 110, 5238 (1999). 